\title{
Guest Editorial: Energy Optimization Methods
}

\author{
Yuri Boykov • Fredrik Kahl • Victor Lempitsky • \\ Frank R. Schmidt
}

Published online: 26 June 2013

(C) Springer Science+Business Media New York 2013

The last 20 years have seen an increasing number of computer vision tasks being formulated as energy optimization problems. Such energy-based modeling can integrate multiple image cues and priors, thus making visual processing robust to noise and resilient to some degree of model misspecification. The scale of the resulting optimization problems however often posits them beyond the reach of generic optimization techniques and solvers. This motivates two active research directions, namely finding new more tractable energy formulations for computer vision problems as well as developing new optimization methods that exploit the specific structure of computer vision energy functionals. This issue presents six papers along these two general lines of vision research.

The paper "A Survey and Comparison of Discrete and Continuous Multilabel Segmentation Approaches" considers perhaps the most popular class of low-level computer vision problems concerned with image labeling (also known as multilabel segmentation or image partitioning). Such problems are typically cast either as continuous (functional-driven) or as discrete (Markov Random Field-driven) energy optimiza-

\author{
Y. Boykov (凶) \\ University of Western Ontario, London, Canada \\ e-mail: yuri@csd.uwo.ca \\ F. Kahl \\ Lund University, Lund, Sweden \\ e-mail: fredrik@maths.lth.se \\ V. Lempitsky \\ Skolkovo Institute of Science and Technology, Moscow, Russia \\ e-mail: lempitsky@skoltech.ru \\ F. R. Schmidt \\ University of Freiburg, Freiburg, Germany \\ e-mail: schmidt@cs.uni-freiburg.de
}

tion. Both modeling approaches have been investigated in much detail over the recent years, and the paper provides the review, the experimental comparison and the discussion of the relative merits of the two approaches.

The paper "Discrete and Continuous Models for Partitioning Problems" further investigates the interplay and the difference between discrete-domain and continuous-domain approaches to image partitioning (labeling). The paper analyzes the advantage of continuous methods over the discrete counterparts in terms of their fidelity to continuouslydefined rotation-invariant functionals. While this advantage comes at a significant computational cost, the paper proposes a new computational scheme permitting considerable decrease of the computational time for an important subclass of continuous-domain image partitioning functionals.

The paper "Combinatorial Optimization of the Discretized Multiphase Mumford-Shah Functional" conversely introduces a new discrete-domain reformulation of the functional that has been traditionally formulated in the continuous domain. The paper demonstrates that such discrete reformulation can benefit from the recent advances in quadratic pseudo-boolean optimization, so that the resulting energy permits efficient global optimization (which is challenging for continuous-domain formulations).

The paper "A Variational Approach to Non-Rigid Video Registration with Subspace Constraints" considers non-rigid registration and optical flow estimation for extended video sequences. Unlike many existing papers that deal with these tasks by matching a pair of frames at a time, this paper considers a "one-shot" reconstruction of dense 2D motion trajectories through time for the entire sequence at hand. This reconstruction task is cast as an optimization of an energy that includes a special prior, enforcing the low-rank constraint on the matrix of recovered trajectories. An efficient scheme to perform such optimization is then presented. 
Graph-based mincut/maxflow optimization remains one of the most popular optimization tools in computer vision for many years. Existing mincut/maxflow algorithms are very efficient for large sparse graphs typical for computer visionrelated optimization problems. However, the performance of these algorithms degrades significantly when graphs outgrow the available memory, which is often the case for problems concerned with videos or 3D images. This important case is addressed in the paper "A Distributed Mincut/Maxflow Algorithm Combining Path Augmentation and Push-Relabel", which presents a new mincut/maxflow algorithm that can efficiently handle very large graphs distributed over several computing nodes.

Finally, the paper "Optimization of Robust Loss Functions for Weakly-Labeled Image Taxonomies" considers a different class of optimization problems that arise in high-level computer vision while learning visual classifiers for a very large number of classes. The authors introduce a new energy functional (a learning loss) that is robust to the ambiguity of class annotation that is typical to large-scale classification datasets (such as ImageNet). The optimization of the resulting functional simultaneously optimizes the performance of the classifiers on the training set and resolves the label ambiguity, which results in the improved classification accuracy at test time.

The issue thus contains papers that cover a range of aspects pertaining to the use of energy optimization, with applications spanning both low-level and high-level computer vision and with methods covering both discrete-domain and continuous-domain optimization. It will therefore appeal to a broad audience interested in various computer vision tasks and applications. 